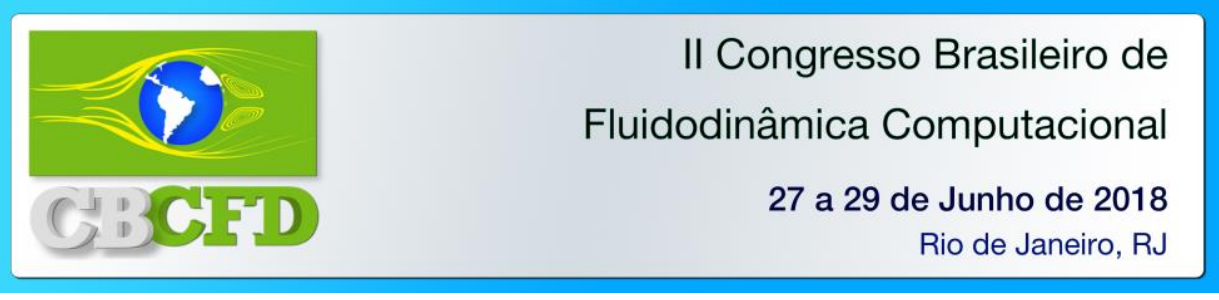

\title{
ANÁLISE NUMÉRICA DO BLOQUEIO DE BOCAL CONVERGENTE VIA FLUIDODINÂMICA COMPUTACIONAL
}

\author{
J. F. T. de CARVALHO ${ }^{1}$, P. S. SCHNEIDER ${ }^{2}$ e L.M. da ROSA ${ }^{3}$ \\ ${ }^{1}$ Universidade Federal do Rio Grande do Sul, PROMEC, Faculdade de Engenharia Mecânica \\ ${ }^{2}$ Universidade Federal do Rio Grande do Sul, PROMEC, Faculdade de Engenharia Mecânica \\ ${ }^{3}$ Universidade Regional de Blumenau, Faculdade de Engenharia Química \\ E-mail para contato: filipe.carvalhosw@ufrgs.br
}

\begin{abstract}
RESUMO - O presente trabalho apresenta um estudo numérico da dinâmica de fluidos dentro de uma turbina Tesla com discos estacionários. A turbina simulada corresponde a um protótipo de escala de laboratório, constituída por um bocal convergente, e as quantidades físicas avaliadas são a velocidade na garganta e na região de jato livre após o bocal e nos canais estreitos entre os discos. Os valores de velocidade na garganta do bocal da turbina são estimados por volta de número de Mach unitário para as condições simuladas.
\end{abstract}

\section{INTRODUÇÃO}

Turbinas de discos múltiplos (MDT) podem ser úteis como um dispositivo de recuperação de energia para fluxos de baixa disponibilidade através da geração de energia elétrica. A MDT foi inventada por Nicola Tesla em 1930, e tornou-se conhecida desde então como turbina Tesla.

A eficiência de uma turbina Tesla pode ser melhorada através da confecção de bocais mais eficientes ou com múltiplos bocais para alimentação do fluido. No presente trabalho a condição de máxima operação do bocal será analisada baseada no equacionamento para uma expansão isentrópica na garganta do bocal (Berchon, 2016).

\section{MODELAGEM MATEMÁTICA}

O escoamento do fluido de trabalho através de bocais convergentes está limitado à condição sônica. A Equação 1 apresenta a razão de vazão mássica por área de passagem na garganta do bocal, para uma expansão isentrópica e limitada ao número de Mach unitário.

$$
\frac{\dot{m}_{t}}{A_{t}}=\frac{P_{1}}{\sqrt{T_{1}}} \sqrt{\frac{k}{R}}\left[\frac{1}{\left[\frac{k+1}{2}\right]^{\frac{k+1}{2(k-1)}}}\right]
$$

O número de Reynolds calculado para a região da garganta do bocal é de 327.787 , o que indica um escoamento turbulento, foi calculado através da seguinte relação: 


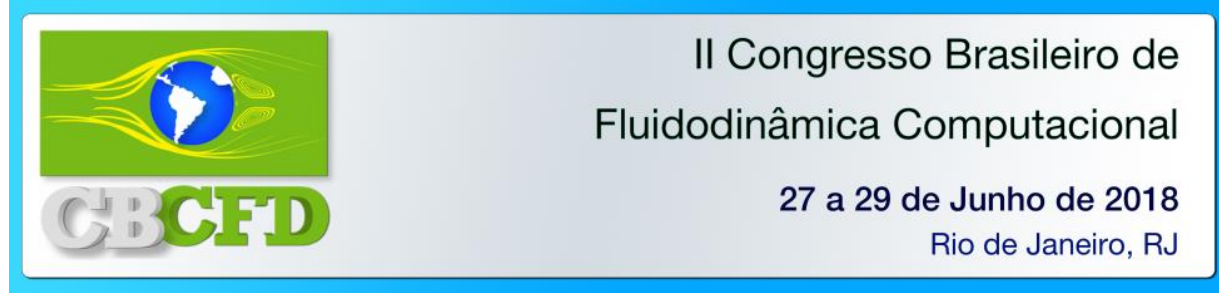

$$
\operatorname{Re}=\frac{U L}{v}
$$

onde $U$ é a velocidade de $309 \mathrm{~m} \cdot \mathrm{s}^{-1}$ na garganta do bocal, $L$ é o comprimento de $20 \mathrm{~mm}$ do bocal, e $v=\frac{\mu}{\rho}$ é a viscosidade cinemática de 1,88 x 10-5 $\mathrm{m}^{2} . \mathrm{s}^{-1}$ (Borgnakke, 2013).

O número de Reynolds calculado para a região do espaçamento de $1 \mathrm{~mm}$ considerando a velocidade média encontrada de $64 \mathrm{~m} . \mathrm{s}^{-1}$ entre os discos é de 3.400, o que sugere um escoamento laminar nessa região.

As condições de contorno adotadas para a presente simulação são apresentados na Tabela 1, para o ar como fluido de trabalho.

Tabela 1 - Condições de contorno da simulação

\begin{tabular}{cccccccc}
\hline $\begin{array}{c}\mathrm{P}(\text { barg) } \\
\text { descarga }\end{array}$ & $\begin{array}{c}\mathrm{T}(\mathrm{K}) \\
\text { descarga }\end{array}$ & $\begin{array}{c}\mathrm{P}(\mathrm{barg}) \\
\text { admissão } \\
\text { bocal }\end{array}$ & $\begin{array}{c}\mathrm{T}(\mathrm{K}) \\
\text { admissão } \\
\text { bocal }\end{array}$ & $\begin{array}{c}\rho\left(\mathrm{kg} / \mathrm{m}^{3)}\right. \\
\text { admissão } \\
\text { bocal }\end{array}$ & $\begin{array}{c}\dot{m}_{\mathrm{ar}} \\
(\mathrm{g} / \mathrm{s})\end{array}$ & $\begin{array}{c}\text { Número de } \\
\text { Reynolds } \\
\text { garganta }\end{array}$ & $\begin{array}{c}\text { Número de } \\
\text { Reynolds } \\
\text { espaçamento } \\
\text { discos }\end{array}$ \\
\hline 0 & 300 & 2,5 & 295 & 3,0 & 47,01 & 330.000 & 3.400 \\
\hline
\end{tabular}

As condições de contorno utilizadas para representar o problema físico consistem em uma vazão mássica na face da admissão da turbina, condição de pressão ambiente na descarga da turbina, e a condição de parede - não deslizamento nas faces dos discos e demais faces da turbina.

O software utilizado foi o Star-CCM+, versão 12.04. Com este, foi simulado um escoamento monofásico, turbulento, considerando fluido compressível. Empregou-se o modelo de escoamento acoplado, em função de ser uma opção mais robusta e precisa no cálculo para altos números de Rayleigh e para escoamentos compressíveis, principalmente na ocorrência de ondas de choque. Embora, quando comparado com o modelo de escoamento segregado, apresente necessidade de maior poder computacional, pois necessita de um uso maior de memória $R A M$, o método acoplado foi utilizado para acoplar os campos de velocidade e pressão, com um esquema de segunda ordem utilizado para interpolar valores para os termos advectivos. As condições de contorno foram definidas para cada subdomínio, composto por um conjunto de faces. O modelo de turbulência RANS Shear Stress Transport (SST) foi empregado, considerando as equações de Navier-Stokes em média temporal. Simulações preliminares foram efetuadas com refinamentos de malha, com 2,00, 4,67 e 7,40 milhões de volumes. Através do método GCI, adotou-se a malha contendo 2 milhões de volumes, a qual apresenta erros abaixo de $1 \%$.

\section{RESULTADOS E DISCUSSÕES}

Resultados obtidos em simulação mostram que o bocal convergente pode levar a velocidades de fluido até a velocidade do som, o que corresponde ao número de Mach unitário. 
O campo de velocidades do fluido paralelo aos discos ao longo de uma seção transversal foi analisado e exibido na Figura 1.

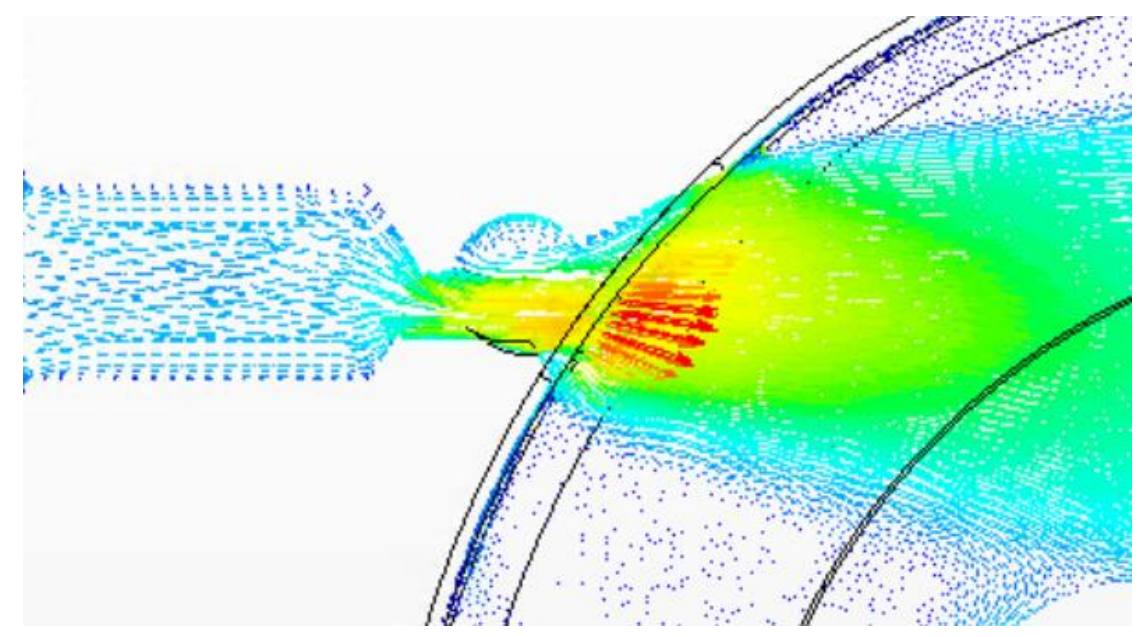

Figura 1 - Campo de velocidade do ar paralelo aos discos posicionada no centro do bocal

O estado do fluido pode ser calculado adotando a hipótese de gás ideal (White, 2010), começando com a velocidade do som c, dado por.

$$
c=\sqrt{\mathrm{kRT}}
$$

Com este valor calculado, o número de Mach obtido foi $M a=0,91$, considerando a velocidade máxima na garganta do bocal de $310 \mathrm{~m} \cdot \mathrm{s}^{-1}$.

Esse valor indica um escoamento praticamente sônico, garantindo a hipótese adotada de escoamento compressível para número de Mach >0,3.

Para verificar os valores na região do espaçamento entre os discos, o campo de velocidades é mostrado no corte transversal ao longo do eixo da turbina na Figura 2.

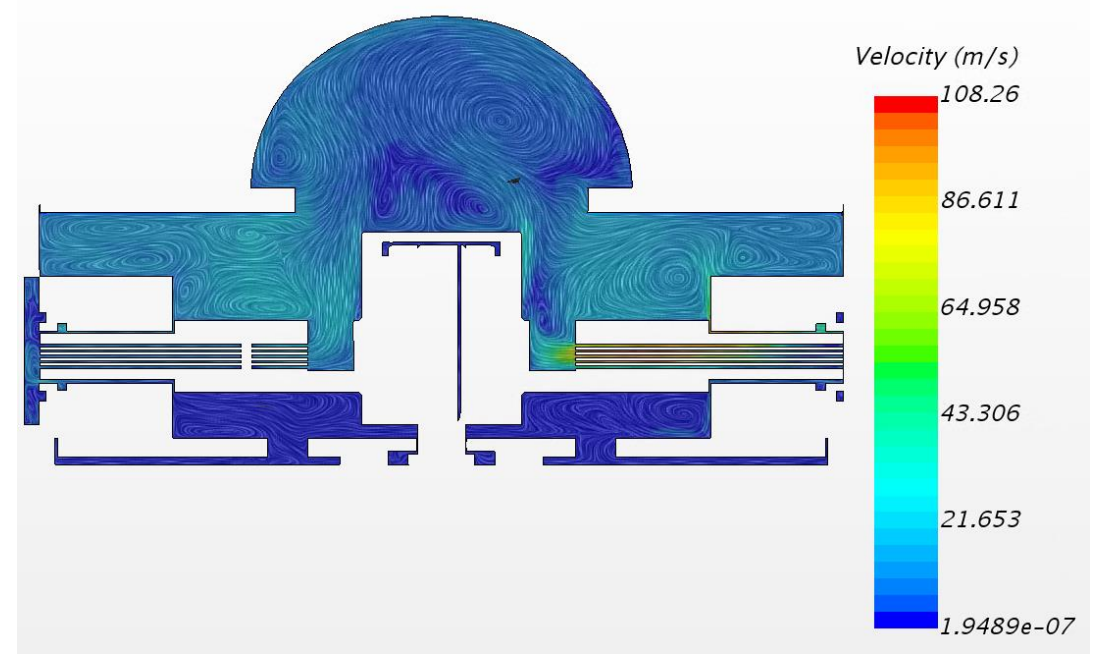

Figura 2 - Campo de velocidades plano eixo central YZ da turbina 


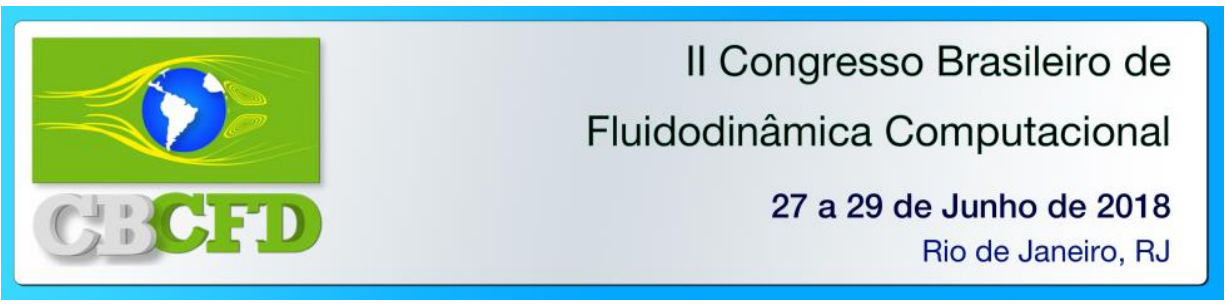

Observa-se que a velocidade máxima encontrada entre os discos foi de aproximadamente $108 \mathrm{~m} \cdot \mathrm{s}^{-1}$, e a velocidade média nesta mesma região variou entre $50 \mathrm{~m} \cdot \mathrm{s}^{-1}$ a $80 \mathrm{~m} \cdot \mathrm{s}^{-1}$. O fluido acelera ao final dos canais estreitos, onde encontra uma região de baixa pressão, sendo este conduzido até os canais de descarga da turbina.

Na Figura 3 são apresentados resultados obtidos para o número de Mach, em um plano na região central do bocal.

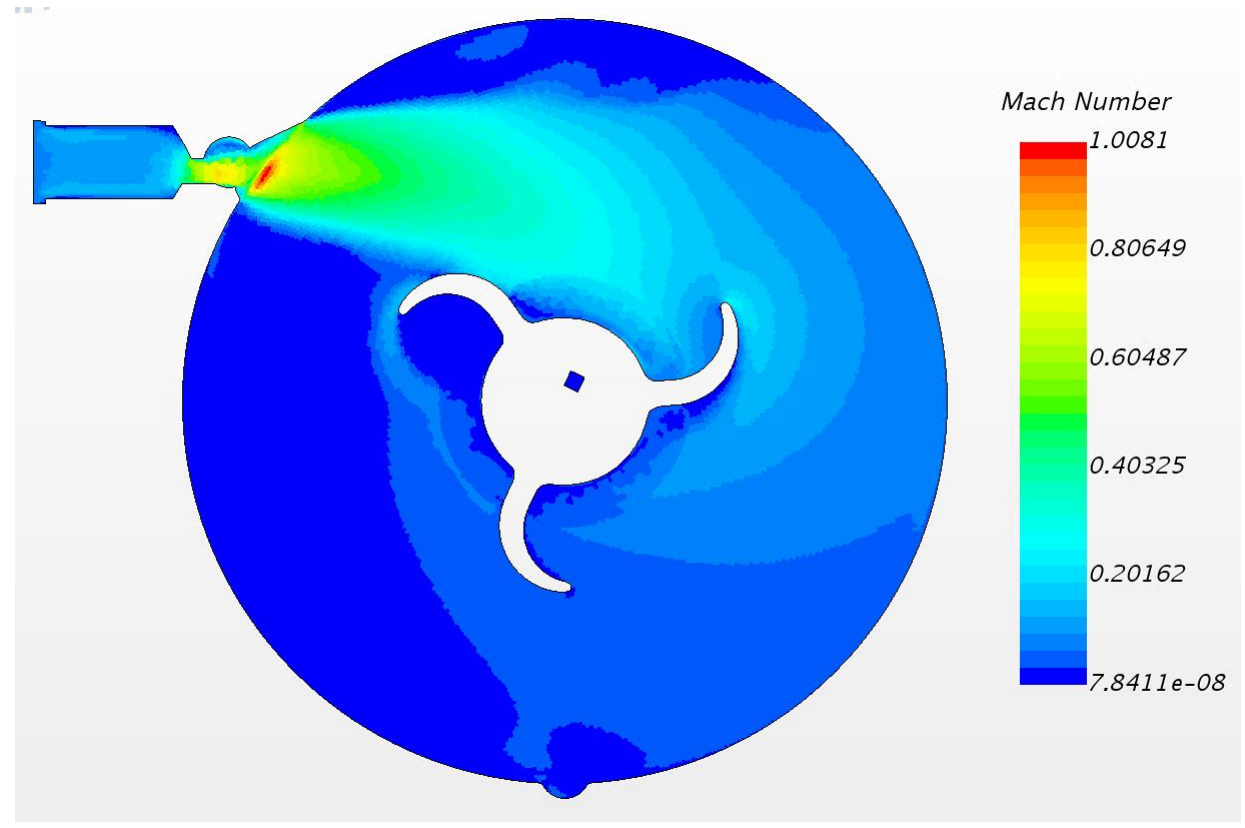

Figura 3 - Número de Mach plano XY posicionado no eixo central da turbina

Observa-se que nesse plano o maior número de Mach chega ao valor unitário. De acordo com a Equação 1, isso sugere que o bocal está operando em sua máxima condição de operação.

Analisando os resultados apresentados acima, observa-se que com as condições de contorno utilizadas, a simulação foi capaz de prever a condição de máxima operação ou bloqueio do bocal analisado.

\section{REFERÊNCIAS}

BORGNAKKE, C.; SONNTAG, R. E.; Fundamentos da Termodinâmica. $8^{\text {th }}$ ed. São Paulo: Blucher, 2013.

BERCHON, L.S. Estudo numérico da influência da geometria de bocais convergentedivergente em escoamentos supersônicos. UFRGS, Porto Alegre, 2016.

WHITE, F. M. Mecânica dos Fluidos. 6th ed. Amgh Editora: Porto Alegre, 2010. 